\title{
Advanced Spaceborne SAR Systems with Array-Fed Reflector Antennas
}

\author{
Sigurd Huber, Marwan Younis, Gerhard Krieger, and Alberto Moreira \\ Microwaves and Radar Institute, German Aerospace Center (DLR), Oberpfaffenhofen, Germany \\ Email: sigurd.huber@dlr.de
}

\begin{abstract}
Since its invention 60 years ago the synthetic aperture radar (SAR) principle has been continuously pushed further in terms of information density. Future SAR systems are required to collect data in different frequency bands and polarizations with finer spatio-temporal resolution and higher quality. These requirements drive technology developments with a trend towards digital radars. This type of radar is highly flexible and configurable compared to its analogous counterpart and allows a relatively easy implementation of digital beamforming (DBF) as well as multiple input multiple output (MIMO) techniques. The demand of highly sensitive SAR sensors enforces antenna concepts with large aperture. A promising candidate are large deployable mesh reflector antennas fed by an array of feed elements. Each feed element, or a subset of feed elements, defines a digital channel to be further processed on board the spacecraft or on ground. These innovative class of high-information content SAR sensors are the result of a carefully optimized design in both, the hardware and the signal processing domain. Here, an advanced SAR sensor concept based on array-fed reflector antennas shall be presented. Space, time and frequency adaptive beamforming techniques which complement the antenna design are introduced and demonstrated by means of numerical simulations.
\end{abstract}

\section{INTRODUCTION}

Radar remote sensing is a technique which has been vastly exploited in spaceborne Earth observation applications in the last decades. With synthetic aperture radar systems information about the reflectivity of distant objects can be retrieved. Those imaging radar systems typically consist of a transmitter which illuminates a certain part of the Earth surface, the footprint, and a receiver which collects the scattered electromagnetic energy. This signal is digitized and downlinked to a ground station.

Future applications like Earth system dynamics monitoring [1] require a short revisit time which in turn rises the need of large swath widths. At the same time operators and investigators are interested in a high information content of the SAR signal. This is traditionally achieved by increasing the signal bandwidth resulting in a higher resolution of the SAR images. Table I compares the performance requirements of a state-of-the-art system like TerraSAR-X with a future SAR system like Tandem-L. Large swath widths and high resolutions are contradicting requirements for conventional SAR systems. Since spaceborne SAR systems demand a very high isolation between the transmit- and receive-channel, they cannot be implemented as continuous wave $(\mathrm{CW})$ radars on a single platform. This technical constraint leaves as only option pulsed radar systems which are subject to a stringent timing of
TABLE I

COMPARISON OF THE PERFORMANCE REQUIREMENTS OF A STATE-OF-THE ART SYSTEM LIKE TERRASAR-X WITH A FUTURE SAR SYSTEM LIKE TANDEM-L.

\begin{tabular}{l|c|c|c}
\multirow{2}{*}{$\begin{array}{l}\text { TerraSAR-X } \\
\text { Tandem-L }\end{array}$} & \multicolumn{3}{|c}{ imaging mode } \\
\cline { 2 - 4 } azimuth resolution & $16 \mathrm{~m}$ & $3 \mathrm{~m}$ & $1 \mathrm{~m}$ \\
& $10 \mathrm{~m}$ & $3 \mathrm{~m}$ & $<1 \mathrm{~m}$ \\
\hline \multirow{2}{*}{ swath width } & $100 \mathrm{~km}$ & $30 \mathrm{~km}$ & $10 \mathrm{~km}$ \\
& $350 \mathrm{~km}$ & $175 \mathrm{~km}$ & $50 \mathrm{~km}$ \\
\hline \multirow{2}{*}{ polarization } & single/dual & single $/$ dual & single $/ \mathrm{dual}$ \\
& quad & quad & quad
\end{tabular}

transmit and receive events. Aiming at high resolutions a broad footprint or, respectively, a broad antenna beam is required. In order to sample the received signal adequately a sufficiently high pulse repetition frequency $(P R F)$ has to be used. The high $P R F$ in turn limits the swath width.

One possibility to overcome this restriction is to transmit a signal using a broad beam and to record with multiple receivers. These individual receiver signals are then processed in order to reconstruct the high resolution image. In terms of system theory, such a SAR configuration would be a single input multiple output (SIMO) system. Classical approaches employ planar array antennas where the aperture is split on receive into multiple subapertures [2]. The performance for a spaceborne SAR system consisting of several formation-flying small satellites and the implications on signal processing have been investigated in [3]. The novel concept of combining a reflector antenna with a feed array, where the signal is digitized almost immediately after the receiver, was first proposed in [4] and further investigated in [5]-[10]. It was demonstrated that such systems have the potential to outperform conventional planar SAR systems. Reflector antenna based systems are already a mature technique for communication satellites. Generally reflector antennas inherently generate a shaped beam due to the mechanical molding of the reflector dish. That means for any given feed position only a certain solid angle can be illuminated. Therefore a set of multiple feed elements is required in order to cover the complete region of interest. By moving the analog to digital converters (ADC) closer to the radio frequency ( $R F$ ) front end it is possible to form beams by means of digital signal processing avoiding a costly analog receiver chain. 


\section{Wide-SWATh SAR System OpERATION}

Spaceborne SAR systems acquire backscatter data in a side-looking geometry in order to avoid ambiguous signal overlap in range. The electromagnetic energy is directed via the transmit beam to the Earth surface. Typically the $3 \mathrm{~dB}$ extent of the footprint in elevation, or range, defines the swath width $X$ (see Fig. 1). The sensor moves in azimuth direction and successively transmits radar pulses. These radar pulses are scattered at the Earth surface and the echo signal is reflected back to the sensor, where it is received and coherently recorded.

A SAR imaging mode, where a receive beam moves over the swath in accordance with the direction of reflection using analog beam-steering, was first suggested in [11] for an array of linear feeds and further developed in [12]-[14] with the innovation of digital receiver hardware. This operation principle, today known as Scan-On-Receive (SCORE), offers the opportunity to map very wide swaths. This SCORE principle

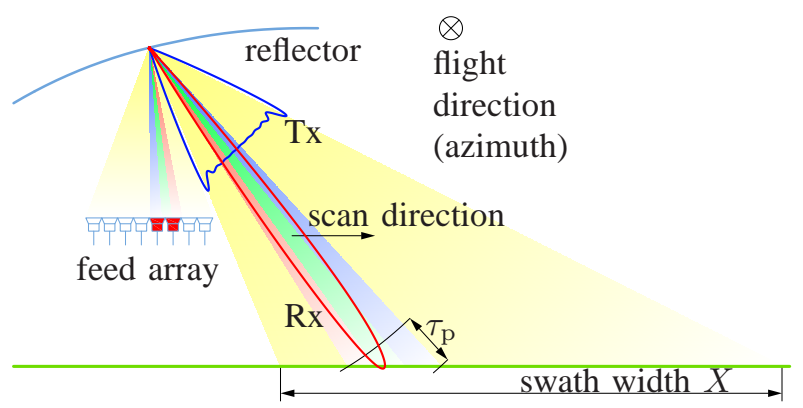

Fig. 1. Reflector system operation with Tx beam illuminating the swath and $\mathrm{Rx}$ beam following the pulse on ground. The Rx beam is steered by activating sets of consecutive feed elements.

has been extended to analog reflector SAR systems by [15], dedicated to the improvement of the performances of SAR systems in terms of 'edge losses' and 'range ambiguities'. In the frame of ultra-wide-swath imaging a reflector SAR system employing a digital feed array is presented for the first time in [4] and further investigated in [7], [16]. The SCORE operation principle of reflector-based SAR systems with linear digital feed arrays is sketched in Fig. 1. The waveform is transmitted with all feed elements active simultaneously, indicated by the yellow pattern. The coverable region on ground depends on the reflector geometry and the size of the feed array. After deflection by the reflector the pulse impinges the ground and moves from the near to the far end of the swath. The signal returning from the ground is again scattered by the reflector and successively collected by different parts of the feed array, indicated by the red feed elements.

\section{A. Limitations}

Analog pulsed SAR systems, specifically single-channel systems, are inherently restricted with respect to their imaging capability. With these systems it is not possible to achieve a large swath width $X$ and a high azimuth resolution at the same time [17]. A high resolution requires a broad azimuth beam, which needs a large $P R F$ in order to sample adequately. However, the high sampling rate constrains the achievable swath width.

Conventional SAR systems with high gain antennas suffer from another trade-off. This trade-off exists between the pulse duration $\tau_{\mathrm{p}}$ and the elevation half-power beamwidth on receive. With long pulses the sensitivity of a SAR system can be increased, allowing to relax the requirements on costly hardware components, like high power amplifiers (HPA). However, the Rx beam requires a width proportional to pulse projected on ground (see Fig. 1). If this condition is violated, that is for very narrow $\mathrm{Rx}$ beams compared to the pulse length, spectral parts are lost involving a degraded range resolution. Additionally, the loss of signal energy results in a reduced SAR image $S N R$. One option to mitigate this effect is to use a broader low gain Rx pattern, which covers the complete pulse projected on ground. The drawback is again a loss of sensitivity.

\section{Multi-Channel Reflector SAR Systems}

The motivation of multi-channel SAR systems is to overcome these afore mentioned restrictions. However, this is not the only reason to deal with multi-channel systems. One could argue, a single-channel system can map an area several times, each covering a different Doppler sub-band, with a low $P R F$ in order to cover a large swath. These multiple acquisitions could then be combined to a single high azimuth resolution image. Evidently, this acquisition concept is prone to temporal decorrelation, especially in vegetated areas and moreover it is time consuming. A far more powerful and flexible way to collect SAR data is to use a multi-channel system, realized on a single platform or via multiple platform transmitters and receivers. Here, the term channel refers to digital signal channels, available as individual data streams, for a digital signal processor. In contrast to conventional SAR systems, arbitrarily many beams can be realized at a single

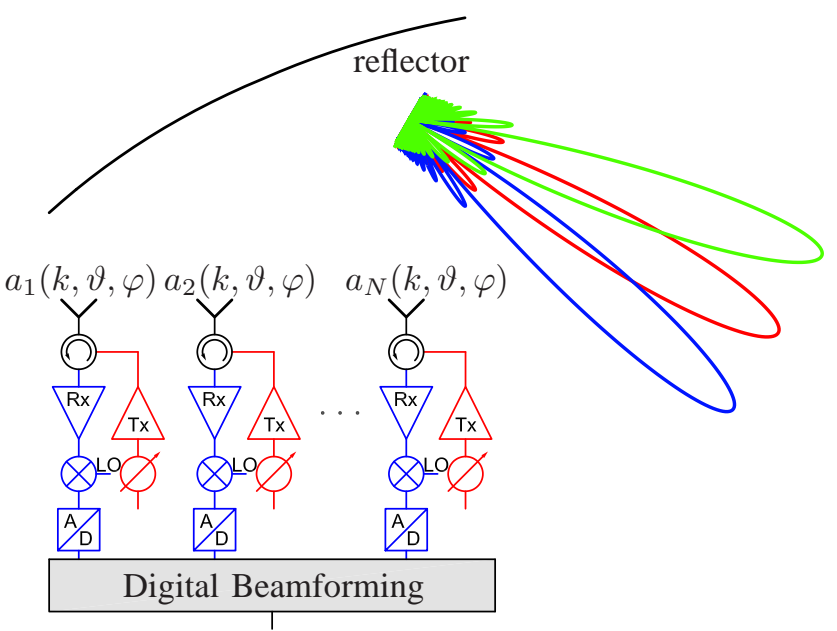

Fig. 2. Hardware concept of a multi-channel reflector SAR system. The A/Dconverters are close to the front end.

time instance, as sketched in Fig. 2. The key idea of digital 
beamforming systems is to move the $\mathrm{A} / \mathrm{D}$ converter closer to the RF frontend. The individual digital channel signals can then either be downlinked to a ground station or directly processed on board the satellite in the digital beamforming unit, as indicated in Fig. 2. Physically a channel is characterized by its spatial and temporal behavior. Each channel is associated with an individual pattern function $a_{i}(k, \vartheta, \varphi) \sim E_{i}(k, \vartheta, \varphi)^{1}$. The patterns $a_{i}$ can be conveniently represented with the so called array manifold

$$
\boldsymbol{a}(k, \vartheta, \varphi)=\left[\begin{array}{lll}
a_{1}(k, \vartheta, \varphi) & \cdots & a_{N}(k, \vartheta, \varphi)
\end{array}\right]^{\top},
$$

where $N$ represents the total number of digital channels. Based upon the received signals

$$
\boldsymbol{u}=\left[\begin{array}{llll}
u_{1} & u_{2} & \cdots & u_{N}
\end{array}\right]^{\top}
$$

a signal model according to

$$
\boldsymbol{u}=\boldsymbol{A s}+\boldsymbol{v},
$$

can be formulated ${ }^{2}$. Here, $v$ represents additive white Gaussian noise. The array response matrix

$$
\boldsymbol{A}=\left[\begin{array}{lll}
\boldsymbol{a}\left(k, \vartheta_{1}, \varphi_{1}\right) & \cdots & \boldsymbol{a}\left(k, \vartheta_{N_{\mathrm{d}}}, \varphi_{N_{\mathrm{d}}}\right)
\end{array}\right]
$$

collects $N_{\mathrm{d}}$ complex array manifold vectors $\boldsymbol{a}$, each associated with a certain direction $(\vartheta, \varphi)$. For instance, the direction $\left(\vartheta_{1}, \varphi_{1}\right)$ could be associated with the signal of interest, while the $N_{\mathrm{d}}-1$ other directions represent ambiguities or generally spatial interference to be suppressed. The corresponding signals are combined in the vector

$$
\boldsymbol{s}=\left[\begin{array}{lll}
s_{1}\left(k, \vartheta_{1}, \varphi_{1}\right) & \cdots & s_{N_{\mathrm{d}}}\left(k, \vartheta_{N_{\mathrm{d}}}, \varphi_{N_{\mathrm{d}}}\right)
\end{array}\right]^{\mathrm{T}}
$$

where $[.]^{\top}$ symbolizes transpose.

\section{A. Digital Beamforming in 3-D Time-Wavenumber Space}

The SAR data are collected in so called radar coordinates $(y, t)$, where $t$ is the temporal variable and $y$ represents the sensor position in azimuth. The azimuth dimension is generated by moving the SAR platform on an essentially arbitrary but known trajectory. Spaceborne SAR systems acquire data by orbiting the Earth on a trajectory, which can be assumed to be locally linear. From the beamforming perspective the question arises, whether a combination of raw data channels can be performed in the spatial domain or not. This is clearly not the case, since targets spatially displaced in azimuth direction will respond with different wavenumbers for a single azimuth position of the SAR sensor. This means, the SAR data need to be transformed into the azimuth wavenumber domain before beamforming. Figures $3 \mathrm{a}$ and $\mathrm{b}$ show the transformation step

\footnotetext{
${ }^{1}$ In the context of digital beamforming these patterns shall represent so called embedded patterns, incorporating effects from coupling. Moreover, these patterns represent the patterns after deflection at the reflector, not the feed element patterns.

${ }^{2}$ In reality the right side of equation (3) is an integral expression. However, for the derivation of the DBF concepts it is sufficient to consider point target responses.
}

(a)

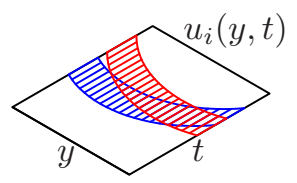

(b)
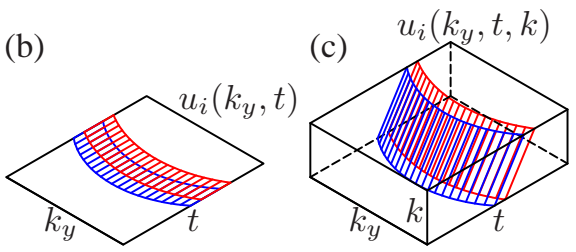

Fig. 3. Time-wavenumber domain beamforming: (a) SAR raw data for two point targets in SAR coordinates azimuth $y$ and range $t$. (b) Fourier transform with respect to azimuth coordinate. (c) Raw data after short-time Fourier transform of range dimension allowing a full separation of individual targets.

of the $i$ th raw data channel $u_{i}(y, t)$ exemplarily for two point targets to the azimuth wavenumber domain $u_{i}\left(k_{y}, t\right)$ (Doppler frequency domain). Here, the targets appear aligned with respect to the wavenumbers. As can be seen in $3 b$ the individual target signals still overlap in the temporal domain. This means in the overlap domain beamforming cannot be applied, since the DBF coefficients can either match to the direction of point target one or to the direction of point target two. A straight forward solution is to expand the temporal domain to the time-frequency domain for instance by a shorttime Fourier transform. This can be done if for instance linear frequency modulated signals, so called chirps, are used. Then the individual targets can be discriminated, as indicated in Fig. 3c, and beamforming techniques can be applied. The

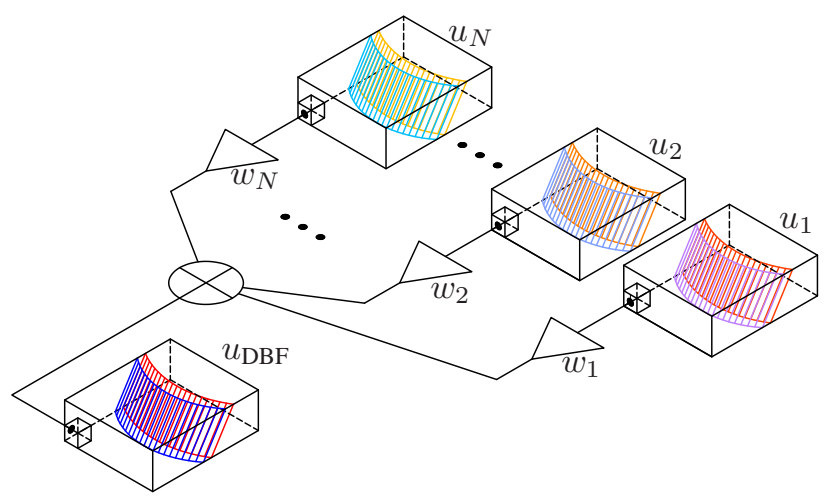

Fig. 4. Digital Beamforming operation, where corresponding samples from the time-wavenumber space $\left(k_{y}, t, k\right)$ are combined by means of complex weights $w_{i}$.

beamforming procedure is then simply a linear combination by means of complex weights of corresponding data samples as shown in Fig. 4. Formally, this can be written as

$$
u_{\mathrm{DBF}}=\boldsymbol{w}^{\top}(k, \vartheta, \varphi) \boldsymbol{u}\left(k_{y}, t, k\right) .
$$

Here, the unique relationship for sidelooking SAR systems geometries between radar coordinates $\left(k_{y}, t, k\right)$ and wavenumber space $(k, \varphi, \vartheta)$ is exploited. It is important to mention that in this context the direction of the signal and the ambiguities is assumed to be known a priori.

\section{Digital Beamforming Techniques}

The core operation in the DBF unit is the combination of the individual received signals $u_{i}$ according to equation (6) 
yielding the beamformer output $u_{\mathrm{DBF}}$. Finding meaningful weights $\boldsymbol{w}$ is a research field on its own, known as pattern synthesis problem. In this context two beamforming concepts shall be considered, which can be derived from a power expression (inserting equation (3) into equation (6)) of the beamformer output according to

$$
\begin{aligned}
P_{\mathrm{DBF}} & =\mathcal{E}\left\{\left|u_{\mathrm{DBF}}\right|^{2}\right\} \\
& =\left|\boldsymbol{w}^{\top} \boldsymbol{A} \boldsymbol{s}\right|^{2}+\boldsymbol{w}^{\top} \mathcal{E}\left\{\boldsymbol{v} \boldsymbol{v}^{\mathrm{H}}\right\} \boldsymbol{w}^{*},
\end{aligned}
$$

with $\mathcal{E}\{$.$\} denoting expectation value, \{.\}^{*}$ conjugate complex and $\{.\}^{\mathrm{H}}$ conjugate complex transpose. Here, statistically independent signal and noise contributions are assumed. The expression $\mathcal{E}\left\{\boldsymbol{v} \boldsymbol{v}^{\mathrm{H}}\right\}$ is known as noise channel covariance matrix $\boldsymbol{R}_{\boldsymbol{v}}$, which might be estimated from samples of the data stream. Then an optimization problem

$$
\begin{array}{cl}
\operatorname{minimize} & \boldsymbol{w}^{\top} \boldsymbol{R}_{\boldsymbol{v}} \boldsymbol{w}^{*} \\
\text { subject to } & \boldsymbol{w}^{\top} \boldsymbol{A}=\boldsymbol{c}
\end{array}
$$

can be formulated, which has the analytic solution

$$
\boldsymbol{w}^{*}=\boldsymbol{R}_{v}^{-1} \boldsymbol{A}\left(\boldsymbol{A}^{\mathrm{H}} \boldsymbol{R}_{v}^{-1} \boldsymbol{A}\right)^{-1} \boldsymbol{c}^{*} .
$$

The vector $\boldsymbol{c}$ is a so called constraint vector, which may be chosen almost arbitrarily, depending on the application. If one recalls the above example, $c$ would have a '1' associated to the direction of interest, and zeros in the directions to be damped. In the literature this solution is known as Linear Constraint Minimum Variance (LCMV) beamformer [18]. A special case of this beamformer is the Minimum Variance Distortionless Response (MVDR) beamformer, that is obtained for $N_{\mathrm{d}}=1$, giving

$$
\boldsymbol{w}^{*}=\frac{c^{*} \boldsymbol{R}_{v}^{-1} \boldsymbol{a}}{\boldsymbol{a}^{\mathrm{H}} \boldsymbol{R}_{v}^{-1} \boldsymbol{a}} .
$$

This means the array response matrix $\boldsymbol{A}$ reduces to a single array manifold vector $\boldsymbol{a}$. Therefore, MVDR beamforming can be understood as a spatial matched filter and as such optimizes the gain or equivalently the signal-to-noise ratio (SNR) in the respective direction of interest.

\section{Digital Beamforming for Spaceborne SAR SENSORS}

The digital beamforming concept introduced in the above sections represents a framework applicable to SAR imaging with multichannel systems in general. However, such an approach is computationally highly inefficient and involves huge amounts of SAR data to be transferred from the spacecraft to ground. A practical approach to this problem is to separate the digital beamforming in elevation and azimuth. Figure 5 shows a 2-D feed array where the individual elements in elevation form a single azimuth channel $u_{\mathrm{az}, j}$ to be downlinked. The beamforming in azimuth can then be performed on ground. Here, the data rate drops by a factor proportional to the number of feed elements in elevation. This means on board the spacecraft a digital hardware for the combination of the individual channels, which performs the spectral discrimination as well
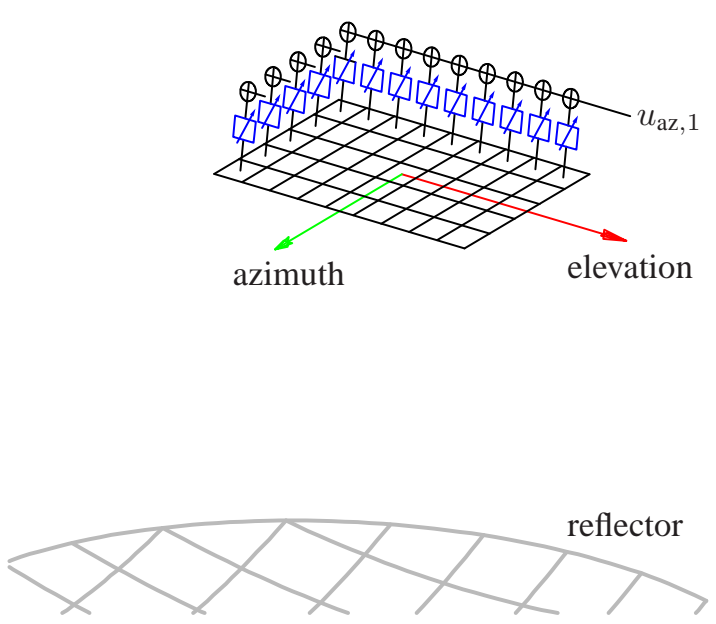

Fig. 5. 2-D feed array concept, where the beamforming in elevation is performed on board the spacecraft. The individual azimuth channels are downlinked for further processing on ground.

as a proper weighting of the signals, has to be implemented. The concepts for beamforming in elevation and azimuth shall be outlined in the next sections.

\section{A. Digital Beamforming in Elevation}

For pulsed radar systems the time interval between two succeeding pulses is $P R I=P R F^{-1}$. Consequently, all digital signal processing in elevation takes place in a time smaller than this interval, which is in the order of several hundred microseconds for spaceborne SAR systems. Even for simple beamforming algorithms the computational power required may exceed those of software based processors. Therefore, signal processing strategies are required which incorporate the concepts derived in the previous sections, but offer enough simplicity to be realized on hardware signal processors implemented on FPGAs or ASICs.

For clarity reasons, in the following derivations the azimuth variable $y$ as well as the azimuth channel index variable $j$ shall be dropped. Formally, the beamforming operation in elevation can be stated as

$$
u_{\mathrm{az}}(t)=\int_{t^{\prime}} \boldsymbol{u}^{\top}\left(t-t^{\prime}\right) \boldsymbol{h}\left(t, t^{\prime}\right) \mathrm{d} t^{\prime} .
$$

This integral represents a time-variant convolution of the SAR raw data with a time-dependent filter function. The time-variant filter performs the spectral decomposition of the received signal, which is basically time invariant, and a proper weighting of the spectral parts. The weighting is time-variant, since the beam follows the pulse on ground.

On board the spacecraft equation (13) has to be implemented by means of digital finite impulse response (FIR) filters. Then the time discrete $(t \mapsto n)$ beamformer output for the $j$ th azimuth channel writes

$$
u_{\mathrm{az}}(n)=\sum_{n^{\prime}=0}^{N_{\mathrm{coef}}-1} \boldsymbol{u}^{\top}\left(n-n^{\prime}\right) \boldsymbol{h}\left(n, n^{\prime}\right),
$$


with the time-variant digital filter

$$
\boldsymbol{h}\left(n, n^{\prime}\right)=\sum_{m=0}^{M-1} \boldsymbol{w}(n, m) \bar{h}\left(n^{\prime}, m\right),
$$

where $M$ is the total number of sub-bands. This filter concept can be understood as multi-rate filtering approach, where the chirp signal is decomposed into several sub-chirps. Consequently, $\bar{h}\left(n^{\prime}, m\right)$ represents a bandpass filter for the $m$ th subband and $N_{\text {coef }}$ is the number of filter coefficients. Clearly, the variable $m$ is associated with the wavenumber $k, k=2 \pi \mathrm{f} / \mathrm{c}$. This is especially important for reflector SAR systems, where the antenna patterns can vary over the frequency band of interest.

\section{B. Digital Beamforming in Azimuth}

As mentioned in the beginning the purpose of digital beamforming in azimuth is to resolve the conflict between wide swaths and high azimuth resolution. One possibility to overcome this restriction is to transmit a signal using a broad beam and to collect the scattered signal with multiple receivers. The individual signals are then processed in order to reconstruct the high-resolution image. A simple way to implement such a system using a reflector antenna is to extend the digital feed array in the azimuth dimension [4], [5] as sketched in Fig. 5.

Concerning digital signal processing strategies, extensive work has been carried out to investigate this problem in the context of planar array SAR systems [19]. In contrast to reflector SAR systems with digital feed arrays, a peculiarity of planar array DBF systems lies in the fact that, depending on the PRF, the synthetic aperture is sampled non-uniformly. This is due to the fact, that the phase centers in azimuth are displaced, depending on the total antenna length and the number of azimuth channels. Based on a generalization of the sampling theorem [20], a concept was developed in order to form the output of a multi-azimuth-channel planar array SAR system [19].

The azimuth processing principle, introduced here, follows the schematic presented in Fig. 3. The basic operation is to transform the $j$ th azimuth channel $u_{\mathrm{az}, j}$ into the wavenumber domain. Dropping the elevation variables $t$ and $k$, the azimuth wavenumber signal writes

$$
U_{\mathrm{az}, j}\left(k_{y}\right)=\frac{1}{v_{y}} \int_{y=-\infty}^{\infty} u_{\mathrm{az}, j}(y) \mathrm{e}^{-\mathrm{j} k_{y} y} \mathrm{~d} y,
$$

where $v_{y}$ denotes the satellite velocity in azimuth. It is important to mention, that $U_{\mathrm{az}, j}$ is a bandpass signal. Consequently, the individual azimuth signals need to be reconstructed on a common finely sampled wavenumber grid prior to beamforming. The second step in the azimuth processing is the combination of the individual channel spectra $\boldsymbol{U}_{\mathrm{az}}\left(k_{y}\right)$ by means of weights according to

$$
U_{\mathrm{DBF}}\left(k_{y}\right)=\boldsymbol{w}_{\mathrm{az}}^{\top}\left(k_{y}\right) \boldsymbol{U}_{\mathrm{az}}\left(k_{y}\right) .
$$

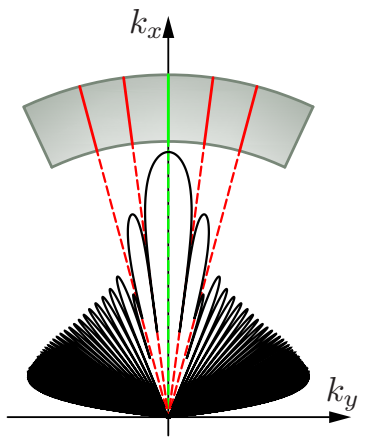

Fig. 6. LCMV principle applied in the wavenumber domain. The LCMV beam is computed such that the signal of interest, indicated in green, is amplified, while ambiguous wavenumbers, marked in red, are damped.

Figure 6 shows an example of an LCMV beam in the direction of the signal (green). The so called azimuth ambiguities, marked by red lines, are suppressed.

With this $U_{\mathrm{DBF}}$ represents the final SAR raw data set after digital beamforming. The beamforming techniques applied shall guarantee a high sensitivity and a good ambiguity suppression. This raw data set can then be processed by conventional SAR focusing routines.

\section{NumERiCAl Simulations}

In this section numerical simulation examples of the above digital beamforming concepts shall be provided. For this a reflector antenna with a diameter of $15 \mathrm{~m}$, fed by an array comprising 36 elevation channels and three azimuth channels, is assumed. The SAR antenna is designed to map a $350 \mathrm{~km}$ swath in a orbit of $745 \mathrm{~km}$.

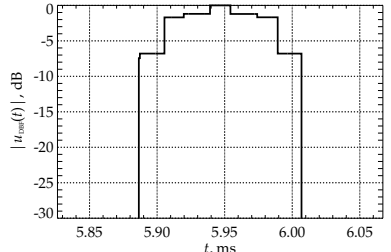

(a)

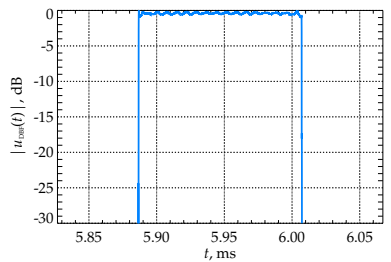

(c)

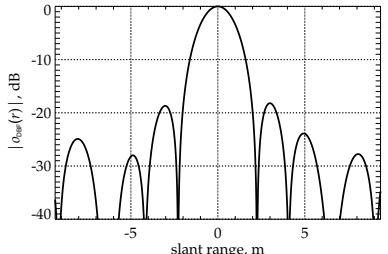

(b)

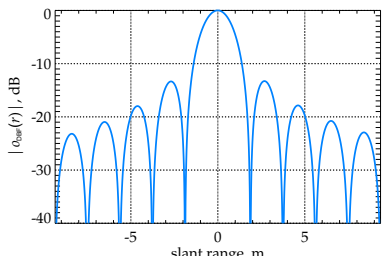

(d)
Fig. 7. Elevation simulation: (a) SAR raw signal without FIR filtering and simple on/off switching of elevation channels. (b) Corresponding range compressed SAR signal. (c) SAR raw signal after MVDR beamforming using a FIR filter with $M=18$ sub bands and 61 filter coefficients. (d) Corresponding range compressed SAR signal.

The first example is an elevation simulation of a point target at swath center. Here, at any time instance three elevation 
channels are active simultaneously. In Fig. 7a the normalized point target signal output in time domain of the central azimuth channel after digital beamforming in elevation is shown. This simulation would represent a conventional case, where no sophisticated frequency adaptive DBF techniques have been applied. The beam is steered to the pulse center, where always sets of three elevation elements, depending on the signal direction, are activated. As can be observed this results in discontinuities in the signal envelope. Correspondingly, Fig. $7 \mathrm{~b}$ shows the range compressed SAR signal. In the lower row simulation of Fig. 7, MVDR beamforming using FIR filters with $M=18$ sub bands and 61 coefficients has been used. With this concept it is possible to recover the signal almost undistorted with a $2.7 \mathrm{~dB}$ improved $S N R$.

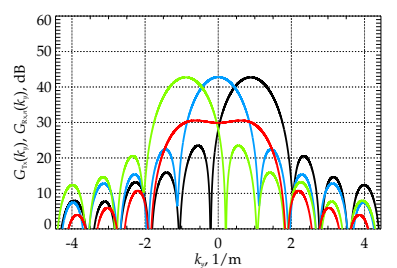

(a)

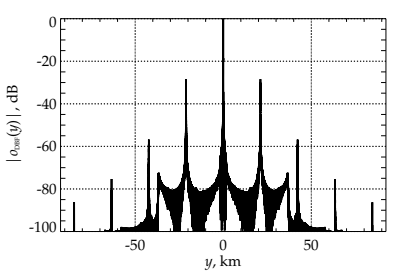

(c)

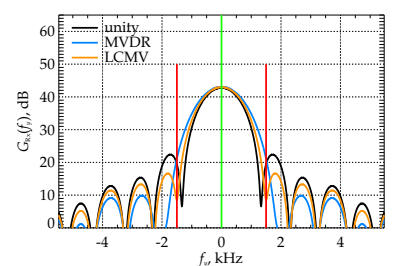

(b)

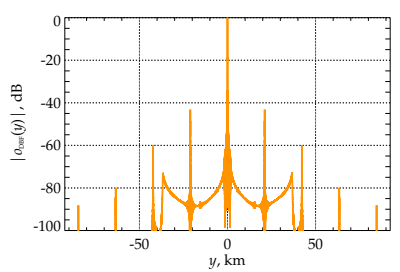

(d)
Fig. 8. Azimuth simulation: (a) Azimuth transmit gain pattern (red curve) and receive patterns versus azimuth wavenumber $k_{y}$. (b) Example of DBF patterns at zero Doppler frequency $f_{y}$ and a PRF of $1.5 \mathrm{kHz}$. (c) SAR impulse response using on/off switching ('unity') and (d) LCMV beamforming.

In a second example digital beamforming in azimuth shall be demonstrated. Figure 8 a shows an azimuth cut of the transmit gain pattern (red curve) and the three patterns corresponding to the three azimuth channels available for beamforming on receive. In Fig. $8 \mathrm{~b}$ an example of patterns after beamforming, pointing at zero Doppler frequency for a PRF of $1.5 \mathrm{kHz}$ is shown. The red lines mark the first left and right azimuth ambiguities. Figures $8 \mathrm{c}$ and $8 \mathrm{~d}$ show the azimuth compressed point target signals using the simple on/off switching technique ('unity') and LCMV beamforming, respectively. Evaluating the azimuth ambiguity suppression one has $-17.8 \mathrm{~dB}$ with simple on/off switching and $-36.6 \mathrm{~dB}$ with LCMV beamforming. Additionally, the on/off technique suffers from a $S N R$ loss in the order of $0.75 \mathrm{~dB}$ compared to LCMV beamforming, since LCMV beamforming uses the power of the three channels more efficiently.

\section{CONCLUSION}

This paper introduces the innovative concept of array-fed reflector SAR systems. In order to meet future performance requirements a generalized framework for digital beamforming with SAR systems has been introduced with the goal of high sensitivity and ambiguity suppression. For spaceborne SAR systems two DBF concepts, in elevation and azimuth, have been discussed. This new generation of future SAR systems has the potential to outperform current state-of-the-art systems significantly.

\section{REFERENCES}

[1] G. Krieger, I. Hajnsek, K. Papathanassiou, M. Eineder, M. Younis, F. D. Zan, S. Huber, P. Lopez-Dekker, P. Prats, M. Werner, Y. Shen, A. Freeman, P. Rosen, S. Hensley, B. Johnson, L. Villeux, B. Grafmüller, R. Werninghaus, R. Bamler, and A. Moreira, "Tandem-L: An Innovative Interferometric and Polarimetric SAR Mission to Monitor Earth System Dynamics with High Resolution," in IEEE International Geoscience and Remote Sensing Symposium (IGARSS), Jul 2010, pp. 253-256.

[2] A. Currie and M. A. Brown, "Wide-swath SAR," IEE Proceedings-F Radar and Signal Processing, vol. 139, no. 2, pp. 122-135, Apr 1992.

[3] Z. Li, Z. Bao, H. Wang, and G. Liao, "Performance Improvement for Constellation SAR using Signal Processing Techniques," IEEE Transactions on Aerospace and Electronic Systems, vol. 42, no. 2, pp. 436-452, Apr 2006.

[4] G. Krieger, N. Gebert, M. Younis, F. Bordoni, A. Patyuchenko, and A. Moreira, "Advanced Concepts for Ultra-Wide-Swath SAR Imaging," in European Conference on Synthetic Aperture Radar (EUSAR), vol. 2, Jun 2008, pp. 31-34.

[5] G. Krieger, N. Gebert, M. Younis, and A. Moreira, "Advanced Synthetic Aperture Radar Based on Digital Beamforming and Waveform Diversity," in IEEE Radar Conference (RADAR), May 2008, pp. 767-772.

[6] M. Younis, S. Huber, A. Patyuchenko, F. Bordoni, and G. Krieger, "Performance Comparison of Reflector- and Planar-Antenna based Digital Beam-Forming SAR," International Journal of Antennas and Propagation, vol. 2009, pp. 1-14, Jun 2009.

[7] A. Freeman, G. Krieger, P. Rosen, M. Younis, W. Johnson, S. Huber, R. Jordan, and A. Moreira, "SweepSAR: Beam-forming on Receive using a Reflector-Phased Array Feed Combination for Spaceborne SAR," in IEEE Radar Conference (RadarCon), May 2009.

[8] S. Huber, M. Younis, A. Patyuchenko, and G. Krieger, "A Novel Digital Beam-Forming Concept for Spaceborne Reflector SAR Systems," in European Radar Conference (EuRAD), Oct 2009, pp. 238-241.

[9] — , "Digital Beam Forming Concepts with Application to Spaceborne Reflector SAR Systems," in International Radar Symposium (IRS), Jun 2010, pp. 1-4.

[10] — "Digital Beam Forming Techniques for Spaceborne Reflector SAR Systems," in 8th European Conference on Synthetic Aperture Radar (EUSAR), Jun 2010, pp. 962-965.

[11] J. H. Blythe, "Radar systems," U.S. Patent 4,253,098, Feb, 1981.

[12] M. Suess, B. Grafmüller, and R. Zahn, "A novel high resolution, wide swath SAR," in IEEE International Geoscience and Remote Sensing Symposium (IGARSS), vol. 3, 2001, pp. 1013-1015.

[13] M. Suess and W. Wiesbeck, "Side-looking Synthetic Aperture Radar System,” European Patent EP 1241487 B1, Sep, 2002.

[14] T. Fujisaka and Y. Oh-Hashi, "Synthetic Aperture Radar System," U.S. Patent 5,059,966, Oct, 1991

[15] J. T. Kare, "Moving receive beam method and apparatus for synthetic aperture radar," U.S. Patent US 6,175,326 B1, Jan, 2001.

[16] G. Krieger, M. Younis, N. Gebert, S. Huber, F. Bordoni, A. Patyuchenko, and A. Moreira, "Advanced Digital Beamforming Concepts for Future SAR Systems," in IEEE International Geoscience and Remote Sensing Symposium (IGARSS), Jul 2010, pp. 245-248.

[17] A. Freeman, W. T. K. Johnson, B. Huneycutt, R. Jordan, S. Hensley, P. Siqueira, and J. Curlander, "The 'Myth' of the Minimum SAR Antenna Area Constraint," IEEE Transactions on Geoscience and Remote Sensing, vol. 38, no. 1, pp. 320-324, Jan 2000.

[18] H. L. V. Trees, Optimum Array Processing. John Wiley \& Sons, Inc., 2002.

[19] N. Gebert, G. Krieger, and A. Moreira, "Digital Beamforming on Receive: Techniques and Optimization Strategies for High-Resolution Wide-Swath SAR Imaging," IEEE Transactions on Aerospace and Electronic Systems, vol. 45, no. 2, pp. 564-592, Apr 2009. 
[20] A. Papoulis, "Generalized Sampling Expansion," IEEE Transactions on Circuits and Systems, vol. 24, no. 11, pp. 652-654, Nov 1977. 